\title{
Deficiência de glicose-6-fosfato desidrogenase na resistência genética à malária: uma revisão sistemática e metanálise
}

\author{
Glucose-6-phosphate dehydrogenase deficiency in genetic resistance to malaria: a systematic review \\ and meta-analysis
}

Deficiencia de glucosa-6-fosfato deshidrogenasa en la resistencia genética a la malaria: una revisión sistemática e metanálisis

Recebido: 04/02/2022 | Revisado: 10/02/2022 | Aceito: 17/02/2022 | Publicado: 26/02/2022

\author{
Marcelo Cerilo Santos-Filho \\ ORCID: https://orcid.org/0000-0002-4221-2728 \\ Universidade Federal de Sergipe, Brasil \\ E-mail: marcelocirilo@hotmail.com \\ Maria Tairla Viana Gonçalves \\ ORCID: https://orcid.org/0000-0001-5763-3181 \\ Universidade Federal de Sergipe, Brasil \\ E-mail: tairlagoncalves22@gmail.com \\ Karolayne Silva Souza \\ ORCID: https://orcid.org/0000-0003-2627-7385 \\ Universidade Federal de Pernambuco, Brasil \\ E-mail: karolaynes7@hotmail.com \\ Myrela Conceição Santos de Jesus \\ ORCID: https://orcid.org/0000-0002-3819-9065 \\ Universidade Federal Fluminense, Brasil \\ E-mail: myrelaj@id.uff.br \\ Luciane Moreno Storti-Melo \\ ORCID: https://orcid.org/0000-0002-2305-7996 \\ Universidade Federal de Sergipe, Brasil \\ E-mail: stortilu@hotmail.com \\ Erika dos Santos Nunes \\ ORCID: https://orcid.org/0000-0002-9519-1473 \\ Universidade do Estado da Bahia, Brasil \\ E-mail: erika.santosnunes@hotmail.com \\ Katia Cilene da Silva Félix \\ ORCID: https://orcid.org/0000-0003-4791-7177 \\ Centro Universitário do Rio São Francisco, Brasil \\ E-mail: katia.felix@unirios.edu.br
}

\begin{abstract}
Resumo
Vários estudos têm sido conduzidos almejando detectar a variedade de marcadores genéticos associados à resistência malárica. Este trabalho teve como objetivo descrever a relação da deficiência de glicose-6-fosfato desidrogenase na resistência do hospedeiro ao Plasmodium spp. Para isso, realizou-se uma revisão sistemática com metanálise, seguindo as orientações PRISMA. A estratégia de busca foi feita no Medline via Pubmed, Science Direct, Lilacs, Scopus e Google acadêmico. O risco de viés foi utilizado para avaliar cada artigo selecionado através da lista de verificação de avaliação crítica do Instituto Joanna Briggs. Posteriormente, em conjunto, os trabalhos foram avaliados através de Teste de Egger. No total, encontrou-se 638 artigos. Destes, 26 participaram da síntese qualitativa e 15 na quantitativa. Dos 26 estudos, 18 relataram haver relação entre o déficit enzimático e a resistência malária. Quanto aos resultados da metanálise, verificou-se que a deficiência de G6PD pode estar associado à resistência do indivíduo desenvolver a malária, principalmente em asiáticos e africanos. Portanto, os resultados deste artigo poderão auxiliar no desenvolvimento de novas estratégias terapêuticas para esta enfermidade, beneficiando a saúde pública mundial.
\end{abstract}

Palavras-chave: Plasmodium, Polimorfismo genético, Resistência a doença; Saúde pública.

\begin{abstract}
Several studies have been conducted aiming to detect the variety of genetic markers associated with malaria resistance. This work aimed to describe the relationship of glucose-6-phosphate dehydrogenase deficiency in host resistance to Plasmodium spp. To this end, a systematic review with meta-analysis was performed, following PRISMA guidelines. The search strategy was done in Medline via Pubmed, Science Direct, Lilacs, Scopus and Google academic. Risk of bias was used to evaluate each selected article using the Joanna Briggs Institute Critical Appraisal Checklist. Subsequently, together, the papers were evaluated using Egger's Test. In total, 638 articles were
\end{abstract}


found. Of these, 26 participated in the qualitative synthesis and 15 in the quantitative. Of the 26 studies, 18 reported a relationship between enzyme deficit and malaria resistance. As for the results of the meta-analysis, it was found that G6PD deficiency may be associated with an individual's resistance to develop malaria, especially in Asians and Africans. Therefore, the results of this article may help in the development of new therapeutic strategies for this disease, benefiting public health worldwide.

Keywords: Plasmodium; Genetic polymorphism; Disease resistance; Public health.

\section{Resumen}

Se han realizado varios estudios para detectar la variedad de marcadores genéticos asociados a la resistencia a la malaria. Este estudio tuvo como objetivo describir la relación de la deficiencia de glucosa-6-fosfato deshidrogenasa en la resistencia del huésped a Plasmodium spp. Para ello, se realizó una revisión sistemática con meta-análisis, siguiendo las directrices PRISMA. La estrategia de búsqueda se realizó en Medline a través de Pubmed, Science Direct, Lilacs, Scopus y Google academic. El riesgo de sesgo se utilizó para evaluar cada artículo seleccionado mediante la lista de comprobación de la evaluación crítica del Instituto Joanna Briggs. Posteriormente, los trabajos se evaluaron conjuntamente mediante la prueba de Egger. Se encontró un total de 638 artículos. De ellos, 26 participaron en la síntesis cualitativa y 15 en la cuantitativa. De los 26 estudios, 18 informaron de una relación entre el déficit enzimático y la resistencia a la malaria. En cuanto a los resultados del meta-análisis, se encontró que la deficiencia de G6PD puede estar asociada con la resistencia de un individuo a desarrollar malaria, especialmente en asiáticos y africanos. Por lo tanto, los resultados de este artículo pueden ayudar al desarrollo de nuevas estrategias terapéuticas para esta enfermedad, beneficiando a la salud pública en todo el mundo.

Palabras clave: Plasmodium; Polimorfismo genético; Résitance a la enfermedad; Salud pública.

\section{Introdução}

A malária é uma enfermidade potencialmente fatal, que acomete mais de 229 milhões de pessoas em todo o mundo, principalmente as residentes de áreas tropicais e subtropicais causando, em média, 409 mil mortes anuais (WHO, 2019; Brasil, 2020). Os agentes etiológicos desta patologia são parasitos do gênero Plasmodium sp. que são transmitidos através da picada da fêmea do mosquito do genêro Anopheles durante o repasto sanguíneo. As principais espécies que acometem humanos são Plasmodium falciparum Welch, 1987, Plasmodium vivax Grassi; Feletti, 1890, Plasmodium malarie Laveran, 1881; Plasmodium ovale Stephens, 1922, Plasmodium Knowlesi Sinton e Mulligan, 1932, Plasmodium simium e Plasmodium cynolmogi Mayer, 1907. Os indivíduos infectados podem desenvolver náuseas, vômitos, astenia, fadiga, hipertermia, sudorese, cefaleia e em casos graves, o óbito (Brasil, 2019; WHO, 2019). Com isso, vários estudos vêm tentando relacionar variantes alélicas que geram proteção aos quadros clínicos da malária grave, na tentativa de descobrir novas formas de amenizar a sintomatologia e acelerar o processo de cura (Khim et al., 2013, Monteiro et al., 2014, Adukpo et al., 2016).

A resistência à malária faz parte da interação de diferentes fatores reguladores da relação parasito-hospedeiro, como fatores genéticos (mutações e polimorfismos) e imunológicos, fazendo com que os indivíduos desenvolvam imunidade adquirida humoral ou celular (Yuthayong et al., 1993; Veronesi et al., 1999, SMITH et al., 2002; Duarte et al., 2003; Nahrevanian, 2006; Coban et al., 2018). Polimorfismos genéticos são definidos quando ocorre uma mutação com frequência $\geq$ a $1 \%$ na população, sendo considerados importantes biomarcadores de gravidade de diversas patologias como cânceres, leishmaniose e tuberculose (Maulén et al., 2007; Motoori-Fernandes et al., 2019; Júnior et al., 2021). Variantes alélicas relacionadas a expressão de Haemoglobin C (HbC), Haemoglobin E (HbE), Haemoglobin $S$ (HbS), $\alpha$-thalassaemia $(\alpha$-thal), $\beta$ thalassaemia ( $\beta$-thal), Glucose-6-phosphate dehydrogenase (G6PD) e Triggering Receptor Expressed on Myeloid Cells I (TREM-I) têm sido associados com a regulação, expressão e função celular, podendo haver um papel importante na resistência a quadros clínicos da malária grave (Caroline et al., 2013; Adukpo et al., 2016; Arguinano et al., 2017; Bonfim et al., 2017).

A enzima G6PD desempenha a função de prevenir o estresse oxidativo celular, através da via metabólica na produção de Nicotinamide Adenine Dinucleotide Phosphate Hydrogen (NADPH) e glutationa, assegurando a homeostase intracelular (Khim et al., 2013; Monteiro et al., 2014). O déficit dessa enzima, é causado por um dos polimorfismos enzimáticos de eritrócitos mais recorrentes em todo mundo e estima-se que 420 milhões de pessoas principalmente da África, América de Sul e Ásia apresentam essa característica (Chu et al., 2017). Em contrapartida, variantes alélicas que proporcionem o déficit da 
enzima G6PD, possivelmente, confere resistência à malária (Khim et al., 2013, Liang et al., 2019; Yi, 2019). O Plasmodium sp. durante o ciclo eritrocítico, gera um estresse adicional à célula hospedeira, causando a hemólise, provocando na membrana eritrocitária alterações decorrentes da oxidação celular, o que pode levar a destruição da célula de forma mais rápida em pessoas acometidas com deficiência da G6PD. Desse modo dificultando que o parasito consiga se desenvolver completamente nas hemácias (Kotepui et al., 2016; Manjurano et al. 2017, Plewes et al., 2017).

Estudos realizados acerca da relação da deficiência de G6PD e a proteção malárica são divergentes quanto aos seus resultados. Há trabalhos que relatam que o déficit dessa enzima confere resistência contra malária grave (Plewes et al., 2017; Manjurano et al., 2017 e Yi et al., 2019), enquanto outros descartam a possibilidade (Jalloh et al., 2004; Valencia et al., 2016; Outtara et al., 2016). Com isso, vários fatores são importantes para analisar a resistência, incluindo a constituição genética tanto do parasito quanto do hospedeiro, bem como, a idade e condição imunológica do indivíduo acometido. Portanto, este trabalho teve como objetivo avaliar se a deficiência da Glicose-6-fosfato desidrogenase pode estar relacionada à resistência à malária por meio de uma revisão sistemática e metanálise.

\section{Metodologia}

\subsection{Estratégia de busca}

A revisão foi conduzida conforme as recomendações do Preferred Reporting Items for Systematic Reviews and MetaAnalyses - PRISMA (Moher et al., 2009), sendo as informações coletadas no Medical Literature Analysis and Retrieval System Online (MEDLINE) via National Library of Medicine (PubMed), Google Acadêmico, Science Direct, Literatura Científica e Técnica da América Latina e Caribe (LILACS). A pergunta de pesquisa foi estruturada no formato PICO, onde P (População) = Pacientes com malária; I (Intervenção) = Polimorfismos no gene Glicose-6-fosfato desidrogenase; C $($ Comparação $)=$ Pacientes sem malária e O (Desfecho, “outcomes”) = Resistencia à malária. Em todas as plataformas, foram utilizados os seguintes vocabulários dentre os Descritores em Ciências da Saúde (DECs) em combinação com o operador booleano AND: ("Polymorphism” AND “Malaria” AND “Glucose” AND "Disease Resistance”). Os estudos, fornecidos pelas buscas, foram explorados até a última página de cada base de dados. Foram também analisadas as referências dos artigos pleiteados nesse trabalho.

\subsection{Critérios de seleção, extração de dados e seleção dos artigos}

Nesta revisão, incluiu-se estudos de caso-controle ou coorte, que utilizaram os exames diagnósticos preconizados pelo Ministério da Saúde para confimarção da infecção pelo Plasmodium (Brasil, 2009), além de revisões sistemáticas e metanálises. Não houve limitações quanto ao idioma e linha temporal.

Desse modo, foram excluídos artigos com dados secundários, duplicados e que envolvessem pesquisas in vitro ou in vivo. O risco de viés foi avaliado em cada trabalho utilizando a lista de verificação de avaliação crítica do Joanna Briggs Institute (JBI) para estudos de caso-controle e de coorte (JBI, 2020), sendo excluídos os estudos que apresentaram alto ou moderado risco de viés $(\leq 50 \%)$.

A seleção dos estudos foi realizada por dois autores independentes, ambos os indivíduos utilizaram todas as combinações determinadas nas plataformas selecionadas. O processo consistiu em duas etapas, a primeira foi composta pela eliminação de artigos duplicados e análise dos títulos, sendo excluídos os que não estavam relacionados a proposta do trabalho. Enquanto na segunda realizou-se uma análise completa dos artigos, eliminando os que não continham informações sobre dados do quantitativo dos grupos casos e controle e não atendiam aos critérios de elegibilidade. 
Após a seleção dos estudos extraiu-se as seguintes informações: Identificação do estudo (autor, ano, continente), características da amostra (número de pacientes em cada estudo), características da coleta de dados (confirmação diagnóstica por malária).

\subsection{Risco de viés entre os estudos}

Para análise de viés entre os estudos foi realizado teste de Egger, recomendado em metanálises que apresentam maior do que 10 estudos, visto que, se baseiam em estimativa e precisão estatística para sugerir se há a presença de viés de publicação na metanálise (Pereira \& Galvão, 2014).

\subsection{Análise dos dados}

Os dados foram tabulados no Microsoft Excel® (2018) e analisados quanto ao teste diagnóstico, continente e principais resultados. Posteriormente, a metanálise foi realizada no Software R (Versão 1.1.463), utilizando o modelo de efeito fixo (Método de Mantel-Haenszel) para a correlação entre a deficiência da enzima G6PD e a resistência malárica, sendo os resultados apresentados em Odds Ratio (OR) e Intervalos de Confiança (IC) de 95\%. Para verificar a existência de heterogeneidade entre os estudos foi realizado teste $Q$ de Cochran e a estatística $\mathbf{I}^{2}$ de Higgins e Thompson. A fim de se explorar a heterogeneidade, executou-se uma análise de subgrupos por continentes. Todos os dados da metanálise foram sumarizados em gráficos de Forest plot.

\section{Resultados e Discussão}

\subsection{Análise qualitativa}

No total, foram identificados 698 estudos, sendo 44,3\% eliminados na etapa de análise dos títulos por duplicada ou por não atenderem aos critérios de elegibilidade. Dentre os 389 estudos restantes que seguiram para etapa de leitura completa e avaliação dos critérios de elegibilidade, 93,3\% foram excluídos. Quanto à análise do risco de viés de cada estudo, dos 26 estudos elegíveis para síntese qualitativa, 57,7\% apresentaram porcentagem maior que 70\% das respostas “sim” para o formulário de avaliação crítica do JBI, significando que apresentaram baixo risco de viés / alta qualidade metodológica, todavia, 42,3\% estudos obtiveram 50\% das respostas "sim", significando moderado risco de viés / alta qualidade metodológica, estes foram excluídos na síntese quantitativa (Figura 1). 
Figura 1 - Fluxograma da seleção dos estudos para revisão sistemática com metanálise acerca da deficiência de G6PD na resistência genética à malária.

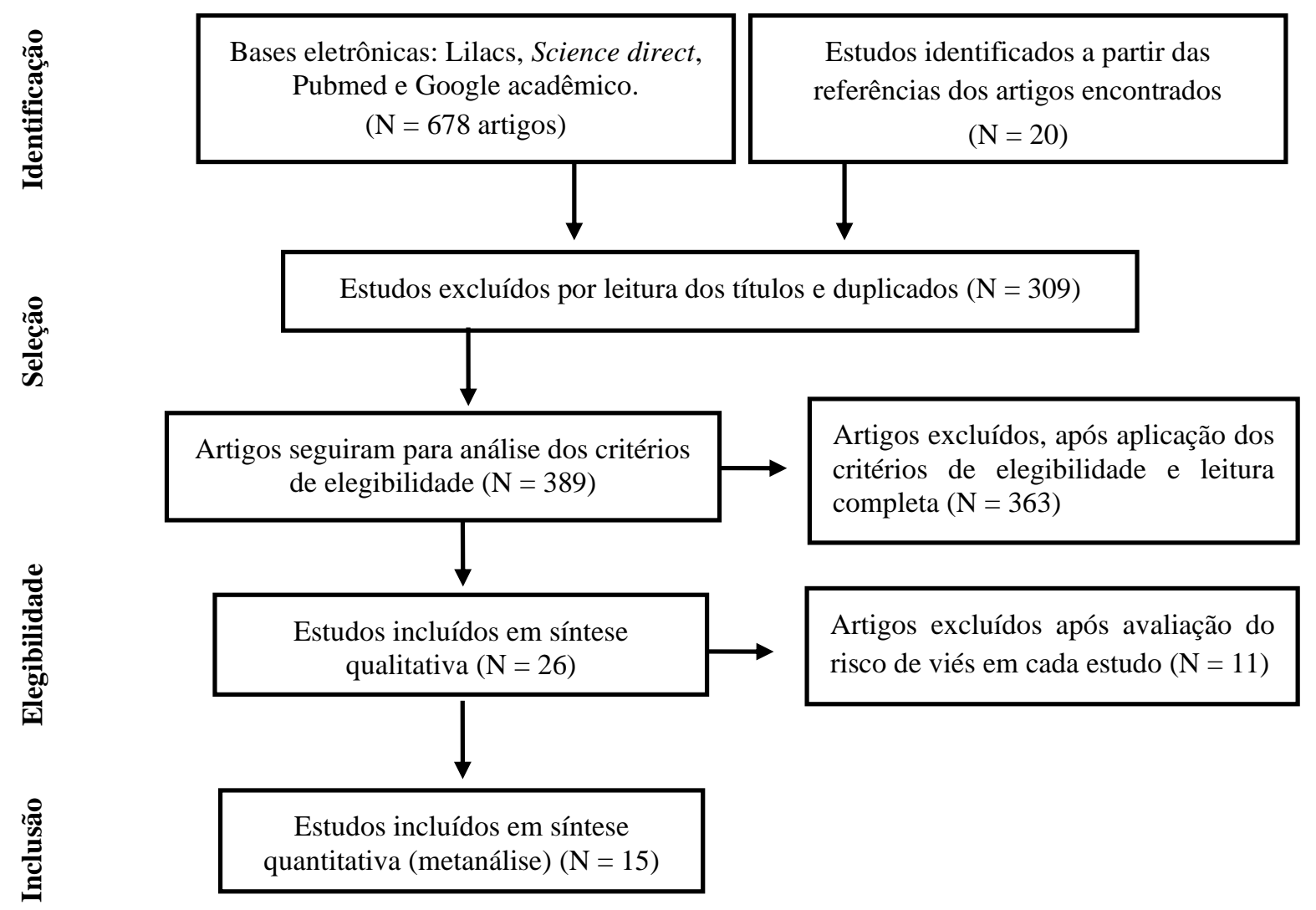

Fonte: Adaptado (Moher et al., 2009).

Os estudos incluídos foram caracterizados de acordo com o método diagnóstico para malária, continente em que a pesquisa foi realizada e os principais resultados (Quadro 1). A maioria dos artigos possuiam indivíduos africanos (53,8\%) e asiáticos $(30,7 \%)$, uma vez que estes continentes são grandes focos do vetor da malária e possuem maiores números de casos da doença (Cavasini et al., 2007; Who, 2019). Nas Américas, principalmente a do Sul, também considerado um continente endêmico para esta patologia, correspondeu a apenas 7,7\% da amostra e na Oceania, apenas 3,8\%, com isso, percebe-se a carência de pesquisas relacionadas a essa temática nesses últimos continentes citados. Todos os trabalhos informaram os critérios utilizados de diagnóstico para os pacientes com malária, entretanto, informações socioeconômico-ambientais não foram descritas pela maior parte dos estudos (73,0\%). Dentre os 26 artigos selecionados, 69,2\% demonstraram haver resistência à infecção por Plasmodium sp. oferecida por polimorfismos que geram deficiência da enzima G6PD. Dentre estes, $5,5 \%$ relataram haver apenas proteção em pessoas heterozigotas e 16,6\% salientam que há resistência somente em homozigotos, 53,8\% dos estudos não apresentaram informação alélica e genotípica dos participantes da pesquisa. Com relação aos 30,8\% dos artigos que afirmaram não haver relação significativa, 25,0\% relataram que o polimorfismo não confere resistência em mulheres heterozigotas e 25,0\% afirmaram não oferecer proteção em homens homozigotos. 
Quadro 1 - Descrição dos estudos incluídos na revisão sistemática e metanálise acerca da deficiência de G6PD na resistência genética à malária.

\begin{tabular}{|c|c|c|c|c|c|c|}
\hline \multirow[t]{2}{*}{ Estudos } & \multirow{2}{*}{$\begin{array}{c}\text { Teste } \\
\text { diagnóstico }\end{array}$} & \multirow[t]{2}{*}{ Continente } & \multicolumn{3}{|c|}{$\begin{array}{c}\text { Deficiência de G6PD Associada } \\
\text { a Resistência Malárica }\end{array}$} & \multirow[t]{2}{*}{$* *$} \\
\hline & & & Sim & Não & Parcial $^{* *}$ & \\
\hline $\begin{array}{l}\text { Alisson et al.* } \\
\text { (1961) }\end{array}$ & Gota espessa & África & & & $X$ & $\begin{array}{l}\text { Apenas em heterozigose em } \\
\text { indivíduos do sexo feminino. }\end{array}$ \\
\hline $\begin{array}{l}\text { Kruatrachue et al.* } \\
\text { (1962) }\end{array}$ & Gota espessa & Ásia & & & $X$ & $\begin{array}{c}\text { Apenas em indivíduos do } \\
\text { sexo feminino. }\end{array}$ \\
\hline $\begin{array}{l}\text { Kruatrachue et al. } \\
\qquad(1966)\end{array}$ & Gota espessa & Ásia & & & $\mathrm{X}$ & $\begin{array}{l}\text { Apenas em homozigose em } \\
\text { indivíduos do sexo feminino. }\end{array}$ \\
\hline $\begin{array}{l}\text { Gilles et al.* } \\
\text { (1967) }\end{array}$ & Gota espessa & África & $X$ & & & \\
\hline $\begin{array}{l}\text { Bienzle et al.* } \\
\quad(1972)\end{array}$ & Gota espessa & África & & & $\mathrm{X}$ & $\begin{array}{l}\text { Apenas em indivíduos do } \\
\text { sexo feminino. }\end{array}$ \\
\hline $\begin{array}{l}\text { Butler et al.* } \\
\text { (1973) }\end{array}$ & Gota espessa & África & $\mathrm{X}$ & & & \\
\hline $\begin{array}{l}\text { Martin et al.* } \\
\text { (1979) }\end{array}$ & Gota espessa & África & & $X$ & & \\
\hline $\begin{array}{c}\text { Barravieira et al.* } \\
\text { (1987) }\end{array}$ & Gota espessa & América & & $X$ & & \\
\hline $\begin{array}{c}\text { Brabin et al. } \\
\text { (1990) }\end{array}$ & Gota espessa & Oceania & $X$ & & & \\
\hline $\begin{array}{l}\text { Kar et al.* } \\
\text { (1992) }\end{array}$ & Gota espessa & Ásia & $X$ & & & \\
\hline $\begin{array}{l}\text { Ruwende et al. } \\
\text { (1995) }\end{array}$ & Gota espessa & África & & & $\mathrm{X}$ & $\begin{array}{l}\text { Apenas em homozigose em } \\
\text { ambos os sexos. }\end{array}$ \\
\hline $\begin{array}{c}\text { Domarle et al*. } \\
\text { (1999) }\end{array}$ & Gota espessa & África & $X$ & & & \\
\hline $\begin{array}{l}\text { Tantular et al. } \\
\text { (1999) }\end{array}$ & Teste rápido & Ásia & & $X$ & & \\
\hline $\begin{array}{l}\text { Jalloh et al.* } \\
\text { (2004) }\end{array}$ & Gota espessa & Ásia & & $X$ & & \\
\hline $\begin{array}{l}\text { Guindo et al. } \\
\text { (2007) }\end{array}$ & Gota espessa & África & & & $X$ & $\begin{array}{c}\text { Apenas em homozigose em } \\
\text { indivíduos do sexo } \\
\text { masculino. }\end{array}$ \\
\hline $\begin{array}{l}\text { Leslie et al.* } \\
\text { (2010) }\end{array}$ & Gota espessa & Ásia & $\mathrm{X}$ & & & \\
\hline $\begin{array}{l}\text { Adinorley et al. } \\
\text { (2011) }\end{array}$ & Gota espessa & África & $\mathrm{X}$ & & & \\
\hline $\begin{array}{l}\text { Francis et al. } \\
\text { (2012) }\end{array}$ & Gota espessa & África & $\mathrm{X}$ & & & \\
\hline $\begin{array}{l}\text { Siguro et al. } \\
\quad(2014)\end{array}$ & Gota espessa & África & $\mathrm{X}$ & & & \\
\hline $\begin{array}{l}\text { Outtara et al. } \\
\qquad(2016)\end{array}$ & Gota espessa & África & & $\mathrm{X}$ & & \\
\hline $\begin{array}{l}\text { Valencia et al. } \\
\text { (2016) }\end{array}$ & Gota espessa & América & & $X$ & & \\
\hline $\begin{array}{l}\text { Dombrowski et al.* } \\
\text { (2017) }\end{array}$ & Gota espessa & América & $\mathrm{X}$ & & & \\
\hline $\begin{array}{l}\text { Plewes et al. } \\
\text { (2017) }\end{array}$ & Gota espessa & Ásia & $\mathrm{X}$ & & & \\
\hline $\begin{array}{l}\text { Manjurano et al.* } \\
\text { (2017) }\end{array}$ & Gota espessa & África & $\mathrm{X}$ & & & \\
\hline $\begin{array}{l}\text { Liang et al*. } \\
\text { (2019) }\end{array}$ & Gota espessa & África & & & $\mathrm{X}$ & $\begin{array}{l}\text { Apenas em homozigose em } \\
\text { ambos os sexos. }\end{array}$ \\
\hline $\begin{array}{l}\text { Yi et al.* } \\
(2019)\end{array}$ & Gota espessa & Ásia & $\mathrm{X}$ & & & \\
\hline
\end{tabular}

Legenda: * Estudos incluídos na análise estatística. ** = Observações dos resultados parciais. Fonte: Autores (2021). 
Ao longo dos tempos, devido a intrínseca coevolução parasito-hospedeiro, uma variabilidade de marcadores genéticos têm sido selecionados por diferentes mecanismos evolutivos entre populações vivendo em regiões endêmicas para malária (Beiguelman et al., 2003; Campino et al., 2006; Cavasini et al., 2007). Assim, polimorfismos que conferem deficiência na enzima G6PD estão entre um dos principais fatores relacionados à resistência ao Plasmodium sp. (Bwayo et al., 2014; Plewes et al., 2017).

Diante da variabilidade genética acumulada nesse marcador, faz-se importante, avaliar quais alelos e genótipos polimórficos para o gene G6PD oferecem resistência a malária. Entretanto, há uma escassez de estudos que demonstrem essa informação, fato evidenciado nesta revisão, no qual a maioria $(72,2 \%)$ dos artigos que afirmaram haver correlação entre polimorfismos no gene G6PD e a resistência malárica não forneceram dados dos genótipos ou alelos.

Embora a perda completa da função da enzima seja presumivelmente letal, mutações que conferem uma deficiência parcial na atividade da enzima são comuns na África, Ásia e América, regiões onde há frequente transmissão do plasmódio (Longo et al., 2002). Existe um grau de conservação em certas regiões do gene que parecem ser essenciais para o desempenho da atividade enzimática e sobrevivência da célula (Notaro et al., 2000). Com isso, mais de 180 polimorfismos que geram deficiência da enzima G6PD e possuem importância clínica, já são descritos na literatura, sendo agrupados em cinco classes de acordo com a atividade normal da enzima, severidade da deficiência, sintomatologia, frequência populacional (Reich et al., 2001; Tishkoff et al., 2002).

Algumas variantes alélicas têm sido mais explicitadas na literatura, tornando-se alvos de estudos entre a relação do déficit de G6PD e resistência malárica, dentre elas a G6PD Mediterrânea (C563T), G6PD A- (A376G/G202A), G6PD A (A376G) e G6PD São Borja (G337A) (Ohanna, 2019). O gene G6PD está ligado ao cromossomo X, assim, indivíduos do sexo masculino têm uma maior probabilidade de apresentar hemólise com repercussão clínica, embora a homozigose ou heterozigose em mulheres com inativação do X distorcido, resulta em alta proporção de cromossomos $\mathrm{X}$ comprometidos e podem ser atingidos da mesma forma (Notaro et al., 2000; Ohanna, 2019).

Estudos afirmam que a deficiência da enzima G6PD só oferece proteção em indivíduos homozigotos (G6PD A-) (Ruwende et al., 1995, Liang et al., 2019). Outros artigos relatam que polimorfismo nesse gene não oferece resistência malárica a homens homozigóticos (Alisson et al., 1961, Kruatrachue et al., 1962). Em contrapartida, na literatura já foi relatado que apenas pessoas do sexo masculino homozigotos são protegidos e mulheres heterozigotas (G6PD A) não, pois como este é um polimorfismo relacionado ao cromossomo X e o homem, possui apenas um, sua proteção será maior do que em mulheres heterozigotas, uma vez que o déficit da enzima será maior, evidenciando uma resistência aumentada (Alisson, 1961, Guindo et al., 2007).

\subsection{Análise quantitativa}

A avaliação de risco de viés entre os estudos, realizado apenas entre os 15 utilizados na metanálise, através do teste de Egger, apresentou baixo risco de viés de publicação, sendo $p=0,22$ (Figura 2). 
Figura 2: Teste de Egger demonstrando o risco de viés entre os estudos da metanálise acerca da deficiência de G6PD na resistência genética à malária.

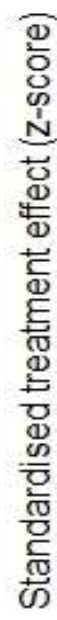

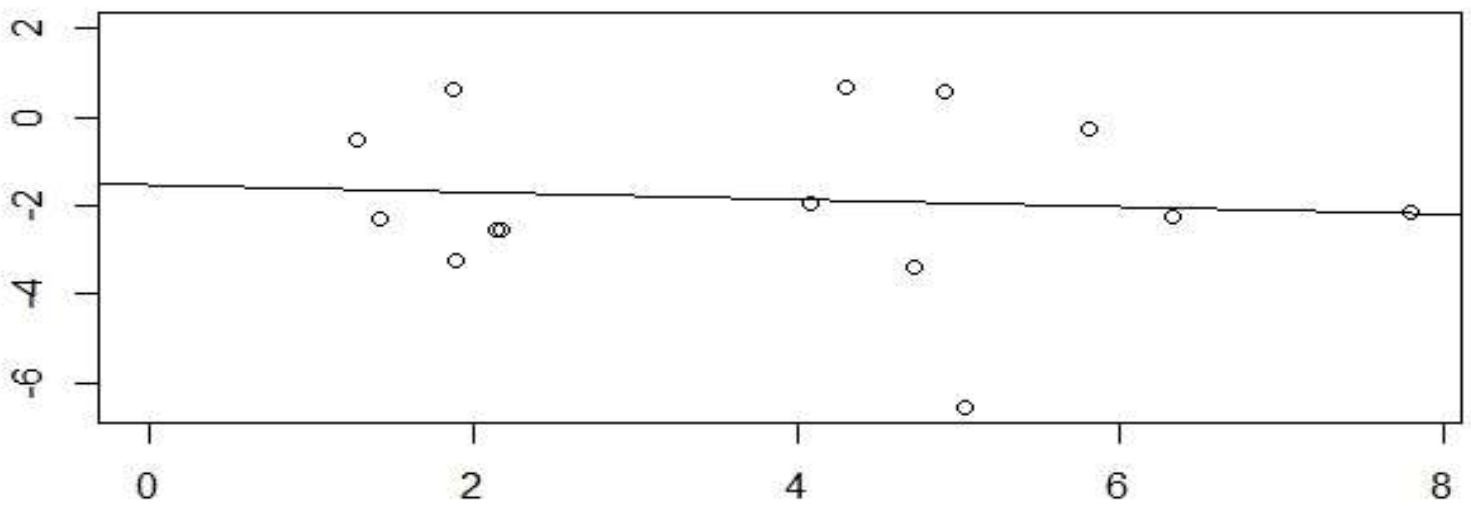

Inverse of standard error

Fonte: Autores (2021).

A metanálise foi realizada pelo efeito do modelo fixo, sumarizando todos os 15 artigos selecionados. Tendo como principais resultados: $\mathrm{OR}=0,70 ;\left(\mathrm{IC} 95 \%, 0.62-0.78\right.$ ); $\mathrm{Q}=p<0,01 ; \mathrm{I}^{2}=81 \%$ (Figura 3).

Figura 3: Gráfico de Forest Plot demonstrando o resultado da metanálise acerca da deficiência de G6PD na resistência genética à malária.

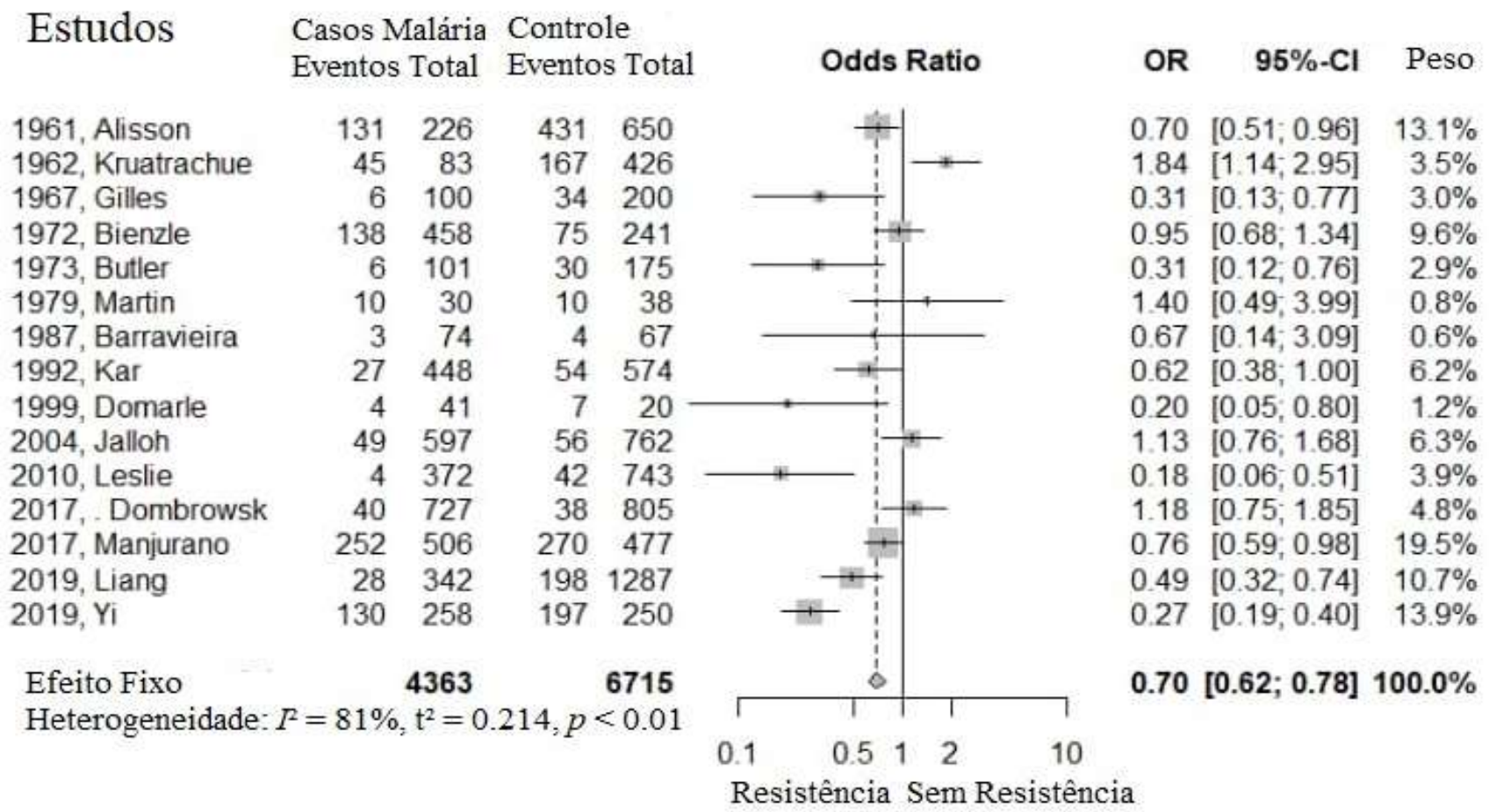

Fonte: Autores (2021).

Dentre os 15 estudos incluídos na metanálise, observou-se associação significativa entre a deficiência de G6PD e a infecção pelo Plasmodium sp. Entretanto, 53,3\% dos trabalhos apresentaram ausência de diferença entre as intervenções. Quanto a resistência, 40,0\% dos artigos confirmaram haver um perfil de resistência à malária associado à deficiência da 
enzima G6PD. Na presente revisão sistemática com metanálise a heterogeneidade foi considerada alta $\left(\mathrm{I}^{2}=81 \%\right), \mathrm{Q}$ de Cochran $(p<0,01)$. Quando há a identificação de alta heterogeneidade entre os estudos, o que geralmente ocorre em estudos genéticos, pode-se realizar várias abordagens, como: ignorar, considerar ou explorar a heterogeneidade (Brasil, 2015). Portanto, decidiu-se explorar a heterogeneidade, optando-se pela análise de subgrupos de acordo com o continente (Figura 4).

Figura 4: Gráfico de Forest Plot demonstrando o resultado da metanálise por continentes acerca da deficiência de G6PD na resistência genética à malária.

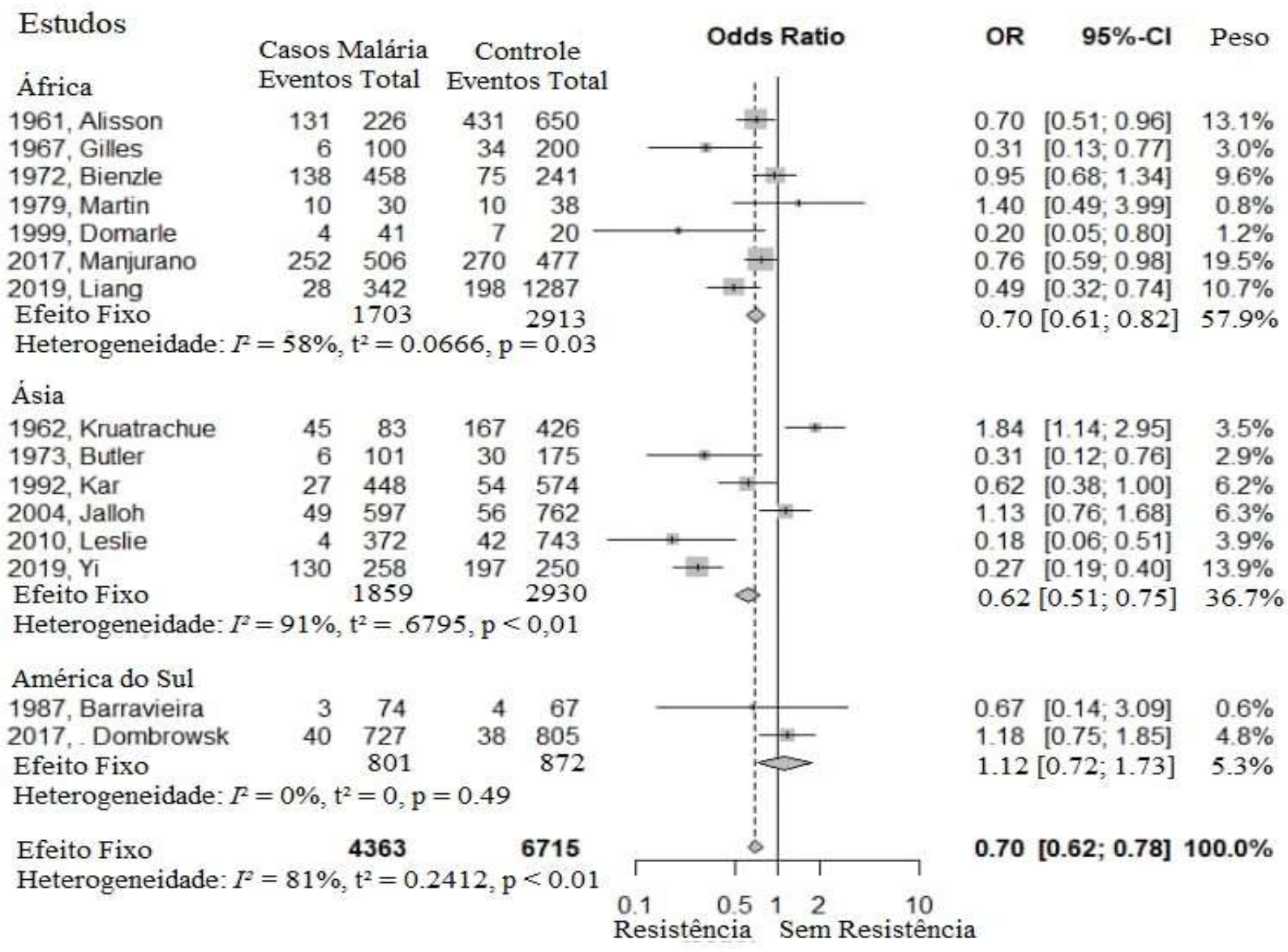

Fonte: Autores (2021).

De acordo com a análise por continente, a associação entre a deficiência de G6PD e a resistência malárica, observouse que na África e Ásia teve positividade, sendo que entre os estudos realizados com indivíduo do continente africano a maioria estabeleceu uma associação de resistência à malária relacionada à deficiência de G6PD, quando se comparado aos asiáticos. No continente americano, verificou-se que o gene estudado não apresentou relação com a resistência malárica.

Esses resultados demonstram a falta de estudos sobre a relação entre a malária e polimorfismos no gene $G 6 P D$ que gerem deficiência da enzima na América, principalmente a do Sul, visto que é um continente abrangente e endêmico para malária (Barravieira et al., 1987). Além disso, a constituição étnica da população gerou uma grande diversidade genética e acrescentando a coevolução parasito-hospedeiro, foram sendo geradas novas variantes alélicas, influenciando fortemente em estudos de associação de marcadores genéticos com perfis de resistência a doenças, podendo explicar a dificuldade em determinar a associação entre a deficiência de G6PD e a resistência malárica nessa região (Dombrowsk et al., 2017). 


\section{Considerações Finais}

A presente revisão sistemática com metanálise evidenciou uma possível associação entre a deficiência da enzima G6PD e a resistência à malária. A análise de subgrupo sugere que dos continentes analisados (África, América do Sul e Ásia), os indivíduos americanos não apresentaram resultados estatisticamente significantes. Portanto, torna-se importante o aumento de pesquisas acerca da relação entre o déficit da G6PD e a resistência malárica, visando estratégias de controle eficazes e novas tecnologias em saúde, como a criação de novos fármacos para o tratamento desta enfermidade.

\section{Referências}

Adukpo, S., Gyan, B. A., Ofori, M. F., Dodoo, D., Velavan, T. P. \& Meyer, C. G. (2016). Triggering receptor expressed on myeloid cells 1 (TREM-1) and cytokine gene variants in complicated and uncomplicated malaria. Trop Med Int Health, 21, 1592-1601.

Allison, A. C., \& Clyde, D. F. (1961). Malaria in African children with deficient erythrocyte glucose-6-phosphate dehydrogenase. British medical jornal. 1, 1346-1349.

Arguinano, A. A., Dadé, S., Stathopoulou, M., Ndiaye, N. C., Xie, T. M. C., Gibot, S. \& Visvikis-Siet, S. (2017). TREM-1 SNP rs2234246 regulates TREM-1 protein and mRNA levels and is associated with plasma levels of L-selectin. PLoS One, 12, 1-15.

Barravieira, B., Meira, D. A., Machado, P. E. A., \& Curi, P. R. (1987). Malária no município de Humaitá, estado do Amazonas. XXI. Prevalência da deficiência de glicose-6-fosfato desidrogenase (G6PD) Em amostra da população e em doentes com malaria causada pelo Plasmodium falciparum. Revista do Instituto de Medicina Tropical. 29.

Bonfim, L. G. S., Magalhães, L. S., Santos-Filho, M. A. A., Peres, N. T. A., Corrêa, C. B., Tanajura, D. M., Silva, A. M., Lipscomb, M. W., Borges, V. M., Jesus, A. R., Almeida, R. P. \& Moura, T. R. (2017). Leishmania infantum Induces the Release of sTREM-1 in Visceral Leishmaniasis. Front Microbiol., 8, 18 .

Eiguelman, B., Alves, F. P., Moura, M. M., \& Nunes A. C. (2003). The association of genetic markers and malaria infection in the Brazilian Western Amazonian region. Memórias do Instituto Oswaldo Cruz, 98, 455-460.

Brasil. Ministério da Saúde (2009). Secretaria de Vigilância em Saúde. Manual de diagnóstico laboratorial da malária.

Brasil. Ministério da Saúde. (2015). Diretrizes metodológicas: elaboração de revisão sistemática e metanálise de ensaios clínicos randomizados.

Brasil. Ministério da Saúde (2019). Secretaria de Vigilância em Saúde. Guia de vigilância em saúde.

Brasil. Ministério da Saúde (2020). Boletim de casos de Malária da Região Amazônica. Situação epidemiológica da malária na região Amazônica, 2018 a 2020 .

Campino, S., Kwiatkowski, D., \& Dessein, A. (2006). Mendelian and complex genetics of susceptibility and resistance to parasitic infections. Seminários em Imunologia. 18, 411-422.

Caroline, L. L. C., Graham, B., John, A. H., Stephen, R., \& Philippe, B. (2013). Monocytes and macrophages in malaria: protection or pathology?. Trends Parasitology. 29, 26-34

Cavasini, C. E., Mattos, L. C., Couto, A. A., Couto, V. S., \& Gollino, Y. (2007). Duffy blood group gene polymorphisms among malaria vivax patients in four areas of the Brazilian Amazon region. Malaria Journal. 6, 1-8.

Coban, C., Lee, M. S. J. \& Ishii, K. J. (2018). Tissue-specific immunopathology during malária infection. Nature Reviews, 18, $266-278$.

Chu; C. S., Bancone, G., Moore, K. A., Win, H. H., Thitipanawan, N., Po, C., \& White, N. J. (2017). Haemolysis in G6PD Heterozygous Females Treated with Primaquine for Plasmodium vivax Malaria: A Nested Cohort in a Trial of Radical Curative Regimens. PLoS Medicine. 4.

Dombrowski, J. G., Souza, R. M., Curry, J., Hinton, L., Silva, N. R. M., Grignard, L., Gonçalves, L. A., Gomes, A. R., Epiphanio, S., Drakeley, C., Huggett, J., Clark, T. G., Campino, S., \& Marinho, C. R. F. (2017). G6PD deficiency alleles in a malaria-endemic region in the Western Brazilian Amazon. Malar Journal, 16.

Duarte, E.C., Pang, L., \& Fontes, C.J.F. (2003). Validade interna de ensaios terapêuticos em malária: análise de estudos de avaliação da emergência de resistência in vivo do Plasmodium vivax a doses padronizadas de primaquina. Revista da Sociedade Brasileira de Medicina Tropical. $36,383-386$.

Guindo, A. Fairhurst, R. M., Doumbo, O. K., Wellems, T. E., \& Diallo, D. A. (2007). deficiency protects hemizygous males but not heterozygous females against severe malaria. PLoS Medicine. 4.

Júnior et al. (2021). TREM1 rs2234237 (Thr25Ser) polymorphism in patientes with cutaneous leishmaniasis caused by Leishmania guyanensis: A case-control study in the State of Amazonas, Brazil. Pathogens, 10(498), 1-11.

Khim, N., Benedet, C., Kim, S., Kheng, S., Siv, S., \& Leang, R. (2013). G6PD deficiency in Plasmodium falciparum and Plasmodium vivax malaria-infected Cambodian patients. Malar Journal. 12.

Kotepui, M., Uthaisar, K., Phunphuech, B., \& Phiwklam, N. (2016). Prevalence and hematological indicators of G6PD deficiency in malaria-infected patients. Infectious Diseases of Poverty. 5. 
Kruatrachue, M., Charoenlarp, P., Chongsuphajaisiddhi, T., \& Harinasuta, C. (1962). Erythrocyte glucose-6-phosphate dehydrogenase and malaria in Thailand. Lancet.2, 1183-1186.

Kruatrachue, M., Bhaibulaya, M., Clongkamnaukorn, K., Harinasuta, C. (1966). Re-examination of the relationship of erythrocyte Glucose-6-Phosphate Dehydrogenase and malaria in Thailand. World Health Organization,

Liang, X. Y., Chen, J. T., Ma, Y., Huang, H., Xie, D., Monte- Nguba, S., Ehapo, C. S., Eyi, U. M., Zheng, Y., \& Liu, X. (2019). Evidence of positively selected G6PD A- allele reduces risk of Plasmodium falciparum infection in African population on Bioko Island. Molecular Genetics Genomic Medicine.8, 111.

Longo, L., Vanegas, O. C., Patel, M., Rosti, V., Li, H., \& Waka, J. (2002). Maternally transmitted severe glucose 6-phosphate dehydrogenase deficiency is an embryonic lethal. EMBO Journal, 21, 4229-4239.

Manjurano, A., Sepulveda, N., Nadjm, B., Mtove, G., Wangai, H., Maxwell, C., Olomi, R., Reyburn, H., Riley, E. M., Drakeley, C. J. \& Clark, T. G. (2017). African glucose-6-phosphate dehydrogenase alleles associated with protection from severe malaria in heterozygous females in Tanzania. PLoS Genet, 11.

Maulén, N. P., \& Cifuentes O. L. (2018). Polimorfismos genéticos asociados a la inmunidad innata y la susceptibilidad genética a la tuberculosis. Revista chilena de enfermedades respiratorias, 34(4), 226-235.

Monteiro, W. M., Val, F. F., Siqueira, A. M., Franca, G. P., Sampaio, V. S., \& Melo, G. C. (2014). G6PD deficiency in Latin America: systematic review on prevalence and variants. Institute Oswaldo Cruz. 109, 553-568.

Moher, D., Liberati, A., Tetzlaff, J., \& Altman, D. G. (2009). The PRISMA Group. Preferred Reporting Items for Systematic Reviews and Meta-Analyses: The PRISMA Statement. PLoS Medicine. 6.

Nahrevanian, H. (2006). Immune effector mechanisms of the nitric oxide pathway in malaria: cytotoxicity versus cytoprotection. Revista Brasileira de Doenças Infecciosas. 10, 283-292

Notaro, R., Afolayan, A., \& Luzzatto, L. (2000). Human mutations in glucose 6-phosphate dehydrogenase reflect evolutionary history. FASEB Journal, $14,485-494$

Ohanna, C. L. B. (2019). Polimorfismos selecionados por patógenos em genes do metabolismo energético e associação com doenças micobacterianas. Tese de doutorado - Instituto Oswaldo Cruz, Pós-Graduação em Bilogia Celular e Molecular, 223

Pereira, M. G., \& Galvão, T. F. (2014). Heterogeneidade e viés de publicação em revisões sistemáticas. Epidemiologia e Serviços de Saúde. 23, 775-778.

Plewes, K., Soontarawira, I., Ghose, A., Bancone, G., Kingston, H. W., Herdman, M. T., Leopold, S. J., Ishioka, H., Faiz, M., Anstey, N., Day, N., Hossain, M., Imwong, M., Dondorp, A., \& Woodrow, C. (2017). Genotypic and phenotypic characterization of G6PD deficiency in bengali adults with severe and uncomplicated malaria. Malar Journal. 16.

Reich, D. E., Reich, D.E., Cargill, M., Cargill, M. \& Bolk, S. (2001). Linkage disequilibrium in the human genome. Nature, 411, $199-204$.

Rocha, A. P., Magalhães, P. K. R., Maia, A. L., \& Maciel, L. M. Z. (2007). Polimorfismos genéticos: implicações na patogênese do carcinoma medular de tireóide. Arquivo brasileiro de endocrinologia e metabologia, 51(5), 723-731.

Ruwende, C., Khoo, S.C., Snow, R. W., Yates, S., Kwiatkowski, D., Gupta, S., Warn P., Allsopp, C., Gilbert, S., Peschu, N., Newbold, C., Greenwood, B., Marsh, K., \& Hill, A. (1995). Natural selection of hemy and heterozygotes for G6PD deficiency in Africa by resistance to severe malaria. Nature. 376, 246249.

Smith, T. G., Ayi, K., Serghides, L., Mcallister, C. D., \& Kain, K. C. (2002). Innate immunity to malaria caused by Plasmodium falciparum. Jornal Oficial da Sociedade Canadense de Investigação Clínica. 25, 262-272.

Joanna Briggs Institute. (2020). Critical Appraisal Tools Checklist. https://jbi.global/critical-appraisal-tools

Tishkoff, S. A. \& Williams, S. M. (2002). Genetic analysis of African populations: Human evolution and complex disease. Nature Reviews Genetics, 3, 611621.

Veronesi, R., \& Focaccia, R. (1999). Imunologia e imunopatologia da malária. Tratado de infectologia.

World Health Organization (WHO). (2019). World Malaria Report. 232.

Yi, H (2019). The glucose-6-phosphate dehydrogenase Mahidol variant protects against uncomplicated Plasmodium vivax infection and reduces disease severity in a Kachin population from northeast Myanmar. Infection. Genetics and Evolution. 75, 1-7.

Yuthavong, Y., \& Wilairat, P. (1993). Protection against malaria by thalassaemia and haemoglobin variants. Parasitol Today. 9, $241-245$. 